\title{
Test of the Constancy of the Velocity of Electro- magnetic Radiation in High Vacuum
}

\author{
Petr Beckmann and Peter Mandics
}

\author{
Department of Electrical Engineering, University of Colorado, Boulder, Colo.
}

(Received October 19, 1964; revised November 23, 1964)

\begin{abstract}
It is pointed out that Einstein's postulate of the constant velocity of light is verified only indirectly by elementary-particle experiments leaning more or less heavily on present electromagnetic theory, the latter being verified only for low velocities. Direct experiments can be explained by the ballistic theory of light if transparent media, such as gases, reradiate as a secondary source. A direct experiment with coherent light reflected from a moving mirror was performed in vacuum better than $10^{-6}$ torr. Its result is consistent with the constant velocity of light.
\end{abstract}

\section{Relativity and Electromagnetism}

Albert Einstein was one of the relatively few people who realized explicitly that his theory rests on the assumption that our present Maxwell-Lorentz electromagnetic theory, experimentally verified only for low velocities of charged matter, will hold also for velocities commensurable with the velocity of light. Considering that our present electrodynamics have grown out of the concept of an elastic ether, whose existence is now disproved beyond reasonable doubt, and that the Maxwell equations do not satisfy the principle of relativity in its simple form using the Galilei transformation, this assumption is far from self-evident. Nor do the verified successes and correct predictions of the Einstein theory (such as increase of mass with velocity, the relativistic Doppler effect, the dilated half-time of mesons, the Moessbauer effect and others) make the above assumption an experimentally verified fact; they prove, as we hope to point out in the following, the consistency of the Maxwell-Lorentz electrodynamics and relativistic mechanics, but not necessarily the universal validity of either.

The Einstein theory assumes the universal validity of the Maxwell-Lorentz electrodynamics; to make them comply with the principle of relativity, it introduces the Lorentz transformation (drastically changing the classical concepts of space, time and mass); it is then found that its results are consistent with all observations made to the present time. But this is not the only possibility. One might possibly also assume the universal validity of the Galilei transformation, leave the concepts of space, time and inertial mass unmodified, and work out electrodynamics that will comply with the Galilei principle of relativity and be consistent with all observations. Except for a single attempt [Ritz, 1908], no such electrodynamics have so far been worked out; but there is no reason to believe that this is inherently impossible. As long as we are only concerned with electrodynamics themselves, or with experiments such as elementary particles (including uncharged ones), where velocities and other quantities are not measured directly, but-at some stage-by interaction with an electromagnetic field, the difference between the above two alternatives is purely philosophical, as they would both yield the same result. But if we perform a direct experiment on the validity of either the Galilei or the Lorentz transformation without in any way relying on electromagnetic theory, we can find out which (if any) of the two transformations is a physical reality; for example, if such a direct experiment lent support to the Galilei transformation, it would expose the Lorentz transformation as a mere equivalence formula making up for inaccurate electrodynamics by suitably deforming space and time to achieve the correct result, but invalid outside the realm of electromagnetic phenomena.

To illustrate the above point, let us assume, for the sake of a counter-example only, that Coulomb's law is inaccurate for high velocities and that it should read

$$
F=\frac{q_{1} q_{2}\left(1-\beta^{2}\right)}{4 \pi \epsilon_{0} r^{2}}
$$

where $q_{1}, q_{2}$ are the electric charges measured in multiples of the charge of an electron, $r$ is the distance between the charges, $4 \pi \epsilon_{0}$ is the constant of proportionality and $\beta=v / c_{0}$ with $v$ the relative velocity of the charges and $c_{0}$ the velocity of light with respect to its source. ${ }^{1}$ For $\beta=0$, (1) would reduce to the orthodox Coulomb law; but there would now be two kinds of charges, a "conservation charge" $q$, measured in integral multiples of an elementary charge (known from nuclear interactions to be conserved and independent of velocity), and a "coulomb charge" $Q$, measured by the force $F=Q_{1} Q_{2} / 4 \pi \epsilon_{0} r^{2}$ exerted by charges on each other. At rest, the two charges will be equal, but for high velocities we have from (1)

$$
Q=q \sqrt{1-\beta^{2}}
$$

${ }^{1}$ Formula (1) is considered for the case $\mathbf{v} \perp \mathbf{r}$; but we wish to state explicitly that we are only making up a counter-example, not putting forward a new theory. 
Writing down the equation of motion of a charged particle with inertial mass $m$, we quickly find it contains the usual ratio $Q / m$, which from (2) now equals (as in the Einstein theory)

$$
\frac{q \sqrt{1-\beta^{2}}}{m}
$$

and no electromagnetic experiment can decide whether the square root in (3) "multiplies $q$ " or "divides $m$ "; i.e., whether (contrary to presently accepted theory) the force on a charge varies with velocity and inertial mass is an invariant, or whether (as in the Einstein theory) charge is an invariant and mass is a function of velocity. ${ }^{2}$

Similarly, it can be shown that the measurements of the half-time of mesons, the relativistic Doppler effect, etc. at some point rely on electromagnetic theory and may permit an alternative interpretation at the expense of present electromagnetic theory. ${ }^{3}$

To resolve this dilemma, we must perform an experiment without relying on any electromagnetic theory at all. Unfortunately, the velocities attained even by rockets in outer space $\left(\beta \approx 10^{-5}\right)$ are still too low to perform direct measurements of length contractions, time dilations and mass increases as given by the Lorentz transformation. However, there remains the possibility of using the velocity of light itself for a test, provided we regard it simply as something moving with the velocity $c_{0}=3 \times 10^{8} \mathrm{~m} / \mathrm{sec}$ with respect to its source and as something periodic (as shown by its capacity to interfere and to produce a Doppler effect), thus not utilizing any of its electromagnetic properties. (By this we mean that we do utilize the capacity of light to interfere, but make no use of the Maxwell equations.) If light is emitted by a source moving with respect to the observer, then according to the Special Theory of Relativity its velocity will remain equal to $c_{0}$ with respect to the same observer, for by the Einstein-Poincaré addition theorem (a direct consequence of the Lorentz transformation),

$$
c=\frac{c_{0}+v}{1+c_{0} v / c_{0}^{2}}=c_{0},
$$

whereas if the Galilei transformation is correct, then

$$
\mathbf{c}=\mathbf{c}_{0}+\mathbf{v}
$$

We do not consider other possibilities, in particular we disregard the ether theory, which does not satisfy the principle of relativity and has been experimentally very thoroughly disproved.

Equation (5), or the "ballistic" theory of light, becomes consistent with practically all experimental evidence if we assume that transparent objects will reradiate the incident light as secondary sources according to the same formula (with their own velocity).

\footnotetext{
${ }^{2}$ In analogy with (2) one might write $M=m \sqrt{1-\beta^{2}}$, with $m$ the invariant inertial mass and $M$ the gravitational mass. (Their equivalence has been demonstrated only for smal B.) Expanding the square root up to second-order terms, the total energy of a body with inertial mass $m$ is then found to be $E=m c^{2}=M c^{2}+\frac{1}{2} m v^{2}$.

${ }^{3}$ Cf. also [Dingle, 1960a, b, c]. [Dingle, 1960c] contains a perplexing paradox on the velocity of propagation of a Doppler effect.
}

This assumption is consistent with the Extinction Theorem of contemporary electromagnetic theory ${ }^{4}$ and moreover fully explains observations made on the spectra of double stars ${ }^{5}$ and in direct laboratory experiments with light [Tolman, 1910, 1912; Majorana, 1918, 1919; Tomaschek, 1924; Bonch-Bruevich and Molchanov, 1956]. In most of the latter, the intent of the experiment was thwarted by a beam splitter or lens which would, by the above hypothesis, reradiate the incident light as secondary sources and thus decelerate it to the velocity $c_{0}$. Moreover, these experiments were made in air at atmospheric pressure, which would have the same effect; this is also true of the one experiment performed before 1962 that was not thwarted by a glass component [Michelson, 1913]. Recently, Kantor [1962] reported an optical experiment performed in air, sharply contradicting (4) and consistent with (5). The experiment measured the fringe shift observed in a Fizeau interferometer, the reflected and transmitted rays being, by hypothesis, accelerated and decelerated respectively by rotating glass windows. However, repetitions of the experiment by Babcock and Bergman [1964] and (in coherent light) by Beckmann and Mandics [1964] indicated that Kantor's result was erroneous, results consistent with (4) being obtained.

\section{Experiments Performed in Vacuum}

The experimental evidence mentioned so far still leaves the ballistic reradiation theory in the field, for the fact that no change in the velocity of light has been observed could be attributed to the presence of air, which might reradiate the incident radiation and act as a secondary source with mean velocity zero. The next logical step to dispose of this argument is therefore to perform a suitable experiment in vacuum. This has been done by Babcock and Bergman [1964], who repeated Kantor's experiment [1962] in vacuum, and by Rotz [1963], who used a three-slit interferometer, keeping two slits stationary and rotating the third, which was covered by glass. Both experiments were consistent with (4) and contradicted (5).

However, since at present it is impossible to obtain a perfect vacuum, we should investigate how high the vacuum must be to prevent possible reradiation by the remaining air molecules. In a perfect vacuum the photons of a light beam would not collide with any molecules at all; we should therefore require that the average number of molecules $N$ in the path of a photon along the whole length $L$ of the interferometer is very much smaller than unity, thus leaving the great majority of photons to travel along the interferometer path in free space without collisions. To estimate $N$, we take it as the average number of molecules in a volume $\lambda^{2} L$, where $\lambda$ is the wavelength of the radiation; thus the cross section of a photon is taken of the order $\lambda^{2}$.

From the kinetic theory of gases, the number of molecules per unit volume of gas is $n=p / k T$, where $p$

\footnotetext{
${ }^{4}$ Cf. [Born and Wolf, 1959]. More simply, one may imagine the incident radiation to
induce conduction or displacement currents which will themselves radiate as secondary induce
sources.

${ }^{5} \mathrm{Cf}$. [Fox, 1962]: for other reasons throwing doubt on the double-star argument cf. [Dingle, 1959].
} 
is the pressure, $T$ is the absolute temperature and $k=1.3805 \times 10^{-23} \mathrm{~J} /{ }^{\circ} \mathrm{K}$ is Boltzmann's constant. Expressing the pressure in torr ( $\mathrm{mm}$ of mercury) and multiplying by $\lambda^{2} L$ we therefore find (for $T=300{ }^{\circ} \mathrm{K}$ )

$$
N=3.21 \times 10^{22} p \lambda^{2} L[\text { molecules; torr } \mathrm{m}, \mathrm{m}] .
$$

TABLE 1. Number of obstructing molecules $\mathrm{N}$ and mean free paths

\begin{tabular}{l|c|c|c|c|c}
\hline \hline \multirow{1}{*}{ Experiment } & $\begin{array}{c}p_{\max } \\
7 u, r\end{array}$ & $\begin{array}{c}\text { Mean } \\
\text { free path } \\
l\end{array}$ & $\begin{array}{c}\text { Mean } \lambda \\
\AA\end{array}$ & $\begin{array}{c}L \\
m\end{array}$ & $\begin{array}{c}N \\
\text { Molecules }\end{array}$ \\
& & & & & \\
& $10^{-2}$ & $5 \mathrm{~mm}$ & 4,740 & 2.76 & $2.0 \times 10^{8}$ \\
Babcock and Bergman [1964] & $1.2 \times 10^{-3}$ & $5 \mathrm{~cm}$ & 6,328 & 0.57 & $8.8 \times 10^{6}$ \\
Rotz [1963] \\
present experiment
\end{tabular}

Table 1 gives the average number of molecules obstructing the path of the interferometer for the above two experiments and for the experiment to be reported below. The corresponding mean free paths are also given for reference. It may be seen from this table that no experiment has so far been performed in sufficiently high vacuum to exclude reradiation completely. By kind permission of Ball Bros., Boulder, Colo., we were able to perform the optical experiment described below in a chamber $15 \mathrm{ft}$ long and $5 \mathrm{ft}$ in diameter in a vacuum of $8 \times 10^{-7}$ torr; as may be seen from table 1 , the present experiment reduces the number of molecules obstructing the interferometer path by more than two orders when compared with previous experiments. Nevertheless, this still leaves an average of some 52,000 molecules that might, by hypothesis, act as a medium reradiating light (the mean velocity of the molecules in the direction of the light beam is zero). Thus the possibility of reradiation by the remaining air molecules even at this low pressure cannot be entirely excluded and this reservation should be borne in mind when drawing conclusions from the present experiment.

The condition for the effect of remanent air molecules to be negligible, i.e., for $N<<1$, is

$$
p \lambda^{2} L<<3.11 \times 10^{-23}\left[\text { torr }, \mathrm{m}^{2}, \mathrm{~m}\right]
$$

For $\lambda=6000 \AA, L=1 \mathrm{~m}$, this leads to $p<<8.6 \times 10^{-11}$ torr, a vacuum approaching the limits of the present state of vacuum technology.

\section{Experimental Setup and Measurements}

To test the constancy of the velocity of light reflected by a moving mirror, the reflected light was passed through a Lloyd interferometer (fig. la) consisting of a slit $\mathrm{S}$ and a Lloyd mirror $\mathrm{A}$. The advantage of this arrangement is that the moving parts of the apparatus are outside the interferometer and hence a fringe shift due to mechanical deformations (simulating a change in the velocity of light) is excluded. The light, by hypothesis (5), reradiated by a moving mirror M with a velocity $c_{0} \pm v$ (where $v$ is the velocity of the mirror $\mathrm{M}$ ), passes through a slit $\mathrm{S}$ and reaches a point
$\mathrm{B}$ on the screen of observation, (a) directly along SB with velocity, $c_{0} \pm v$, and (b) by diffraction along $\mathrm{SA}$ and reflection along $\mathrm{AB}$; the latter being, by hypothesis, due to reradiation by the stationary Lloyd mirror, the velocity of the light along $\mathrm{AB}$ is only $c_{0}$. Thus, the difference in transit times of the direct and the reflected rays will vary with the velocity of $M$ and hence the interference fringes observed on the screen B should shift when $v$ varies. ${ }^{6}$

Let $x$ be the distance of the slit $\mathrm{S}$ or its image $\mathrm{S}^{\prime}$ from the plane of the Lloyd mirror and let $B$ be an arbitrary point on the screen at a distance $y$ from the same plane (fig. la). Let $v / c_{0}=\beta$; then the transit times are

$$
\begin{aligned}
t_{\mathrm{SA}}=\frac{\sqrt{x^{2}+q^{2}}}{c_{0}(1+\beta)}, \quad t_{\mathrm{AB}}=\frac{\sqrt{y^{2}+(L-q)^{2}}}{c_{0}} & , \\
t_{\mathrm{SB}} & =\frac{\sqrt{(x-y)^{2}+L^{2}}}{c_{0}(1+\beta)} .
\end{aligned}
$$

In calculating the transit time difference

$$
\Delta t=t_{\mathrm{SA}}+t_{\mathrm{AB}}-t_{\mathrm{SB}},
$$

${ }^{6}$ The interference fringes in a Lloyd interferometer are formed in exactly the same way as in the case of a radio antenna over a flat earth.

(a)
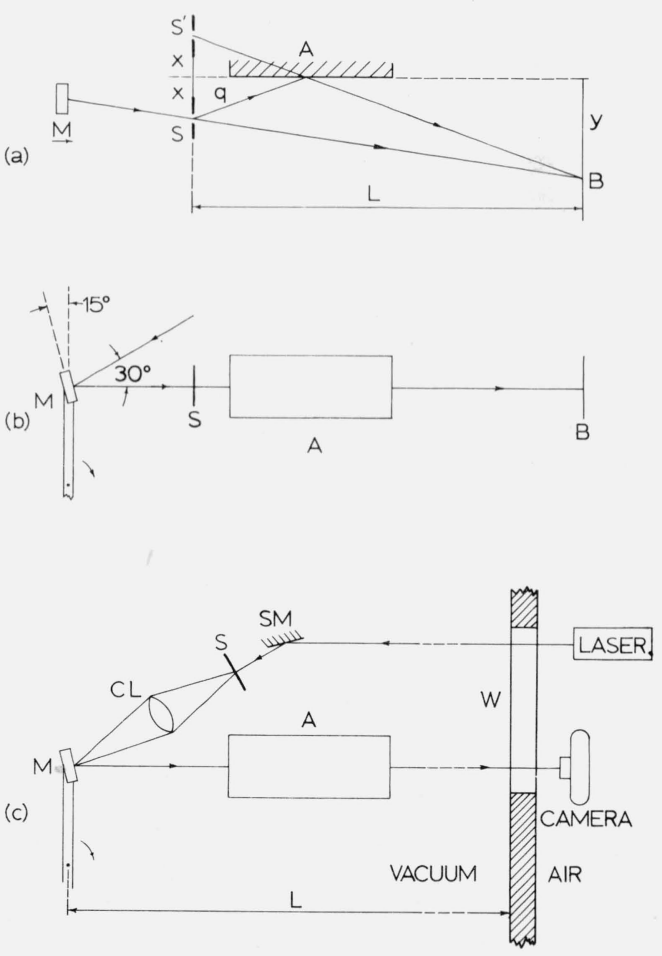

FIGURE 1. Moving mirror and Lloyd interferometer: (a) elevation, (b) plan, (c) plan for arrangement with imaged slit, also showing the arrangement in the vacuum chamber. 
we note from figure la that the distance from the plane of the slit to the point of reflection $\mathrm{A}$ is $q=x L /(x+y)$ and expand the square roots in (7) neglecting 4 th and higher orders in $(x \pm y) / L$. If $\Delta t_{0}$ is the transit-time difference for $v=0$, then we find, neglecting secondorder terms in $\beta$ and since $L \gg x, y$

$$
\Delta t-\Delta t_{0}=\frac{L \beta}{c_{0}} \frac{y}{x+y}\left[1+\frac{(x+y)^{2}}{2 L^{2}}\right] \text {. }
$$

Since $(x+y) / L \ll 1$, the second term in the square brackets may again be neglected and we obtain the relative fringe shift at the point $B$ compared to the stationary case.

$$
\Delta=\frac{c_{0}\left(\Delta t-\Delta t_{0}\right)}{\lambda}=\frac{\beta L}{\lambda} \frac{y}{x+y}
$$

If the velocity of $M$ is reversed from $+v$ to $-v$, the expected shift is thus

$$
\Delta_{ \pm}=\frac{2 \beta L}{\lambda} \frac{1}{1+x / y}
$$

The distance $y$ may be measured on the photograph of the fringes (by utilizing the diffraction fringes due to the edge of the Lloyd mirror); the spacing $s$ between the fringes is also measured from the photograph and it is easily shown that $x=\lambda L / 2 s$. It then follows from (11) that the arrangement will be most sensitive for $x<<y$; i.e., for low-order fringes.

Figure $1 \mathrm{~b}$ shows a schematic plan of the arrangement. The mirror $\mathrm{M}$ was mounted on a rotor driven by an electric motor, the speed of which could be varied and reversed. The plane of this mirror was turned by $15^{\circ}$ from the axis of the rotor as shown so as to accelerate, by hypothesis, the reradiated light by the full component of the circumferential velocity. It was mounted on the rotor at a distance of $12.8 \mathrm{~cm}$ from the axis of rotation.

To remove possible objections that the slit might act as a stationary secondary source and thus reduce both rays to the velocity $c_{0}$, an alternative arrangement was also used (fig. lc): a convex lens CL of focal length 3.5 in. was used to produce a real image of the slit $S$ in space, and the mirror $\mathrm{M}$ moved through this image, throwing its light onto the Lloyd mirror as before. Figure lc also shows the arrangement in the vacuum chamber: the laser beam passed through the window W of the chamber, was reflected by the mirror SM into the slit $\mathrm{S}$ and after traversing the interferometer as described above passed through the same window W into the camera $\mathrm{C}$ outside the chamber. The speed of the motor was measured by a magnetic pickup mounted near the shaft of the motor. The pulses induced in it by two bolts protruding from the motor shaft were counted by a frequency counter. In air, the speed was also measured by illuminating the rotor stroboscopically. A helium-neon gas laser $6328 \AA$, 0.2 to $0.5 \mathrm{~mW}$, beam diameter $2.5 \mathrm{~mm}$, divergence 80 sec of arc) was used as a source of light. Figure 2 shows the entire interferometer and figure 3 a detail of the rotor with the mirror $\mathbf{M}$.

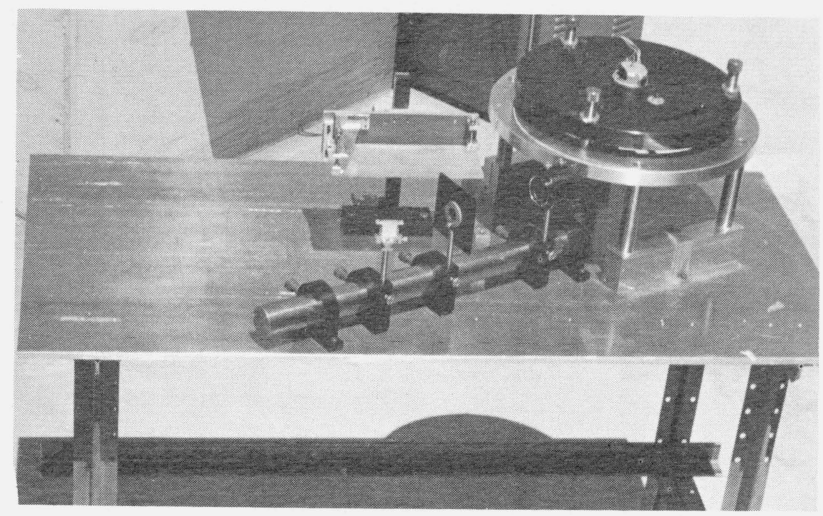

FIGURE 2. View of the interferometer assembly.

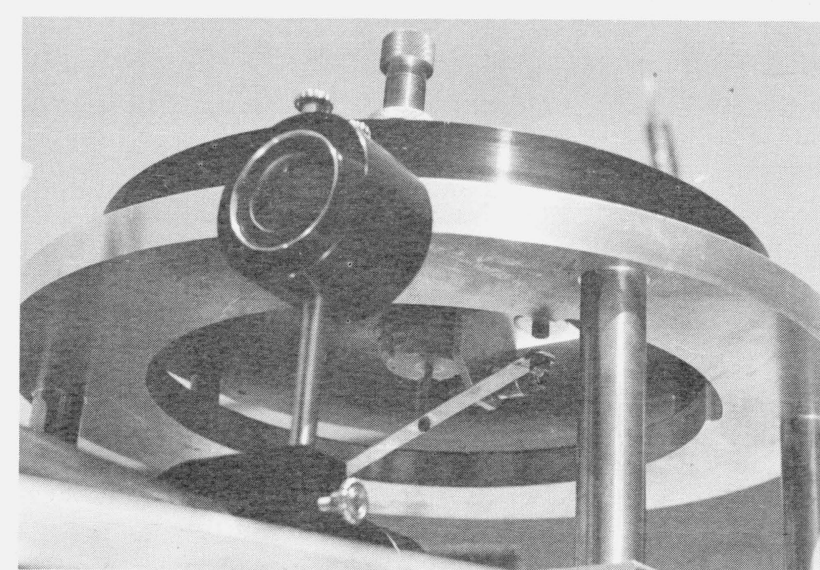

FigurE 3. Detail of motor assembly showing lens CL, rotor wit mirror $M$, and magnetic pick-up.

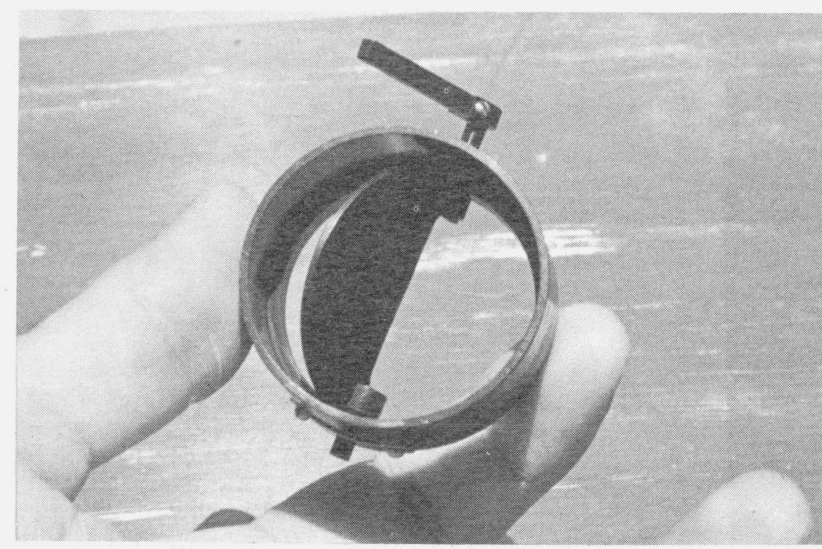

FigURE 4. Semicircular shutter 
Observations were made as follows. The fringes were made to fall directly on the film of a $35 \mathrm{~mm}$ camera with its optics removed. A semi-circular shutter (fig. 4), mounted in place of the optics, could be turned to block one or the other half of the field of view. With the motor running at a certain speed, one-half of the film frame was exposed to the resulting fringes. The motor was then reversed and the shutter turned to expose the other half of the frame to the second set of fringes. The two photographs of fringes were thus exactly opposite each other except for a possible shift as in (11). To make such a shift even more detectable, a narrow strip was cut out of the shutter near its diameter (fig. 4) so that the two exposures slightly overlapped and a strip near the center of the photograph was thus exposed to both sets of fringes (figs. 5, 7). It is estimated that a shift of 0.1 of a fringe would have been readily detected.

The apparatus was provisionally checked in air; both the arrangements of figures $1 \mathrm{~b}$ and $l \mathrm{c}$ were tried. The length $L$ was varied up to $2.1 \mathrm{~m}$, and the motor was made to run up to speeds of $3,400 \mathrm{rpm}$, corresponding to values of $\beta$ up to $1.52 \times 10^{-7}$. The ratio of $x / y$ was of the order of $10^{-4}$, so that the second factor in (11) may be set equal to unity. Thus, if (5) were correct and the air had no effect, the expected fringe shift should, from (11), vary up to $\Delta=1.1$; but in fact no shift was observed (fig. 5). This is consistent with previous experiments [Babcock and Bergman, 1964; Beckmann and Mandics, 1964; Rotz, 1963].

The apparatus was then put in a vacuum chamber with the laser and the camera outside as shown in figure lc. For the arrangement as in figure $1 b$, the vacuum was $10^{-6}$ torr and $L=4.0 \mathrm{~m}$; for the imaged slit as in figure $1 \mathrm{c}$, the vacuum was $7 \times 10^{-7}$ torr and $L=4.25 \mathrm{~m}$. The shift to be expected from (11) under these conditions is plotted in figure 6 ; the points where measurements were taken are indicated by circles no shift was observed. Figure 7 shows a typical picture taken with the interferometer in vacuum.

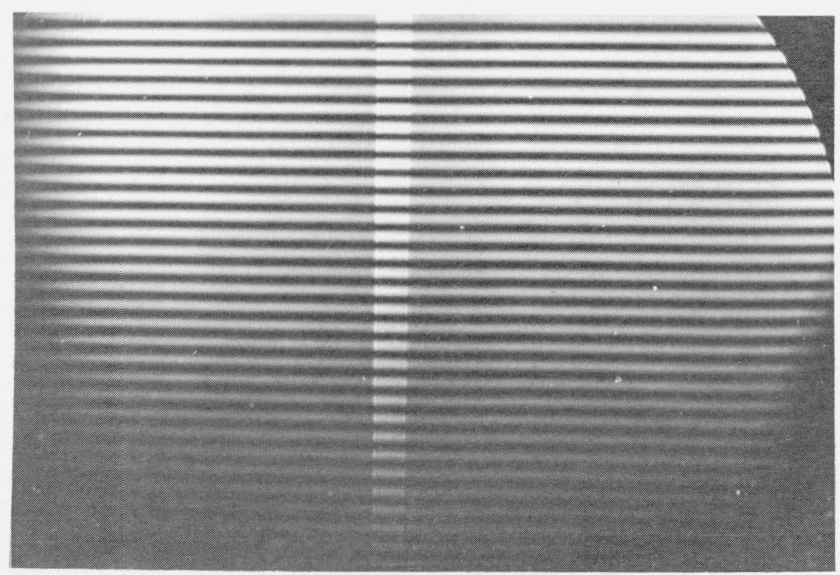

FiguRE 5. Lloyd fringes.

The two halves of the picture correspond to two senses of rotation of the motor. The central strip has been exposed to both sets of fringes.

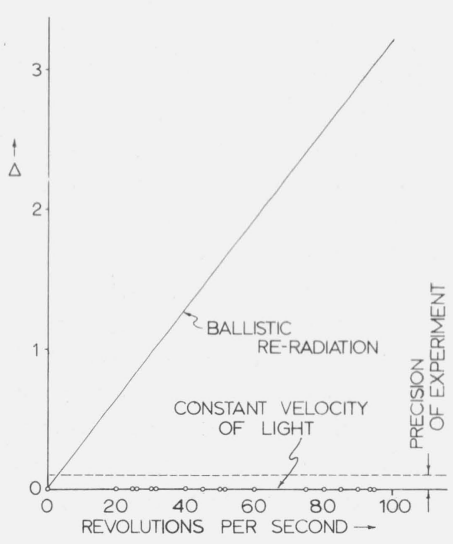

FIGURE 6. Fringe shift predicted by the ballistic theory in the reradiation version and by the special theory of relativity.

Circles indicate motor speeds at which photographs of the fringes were taken.

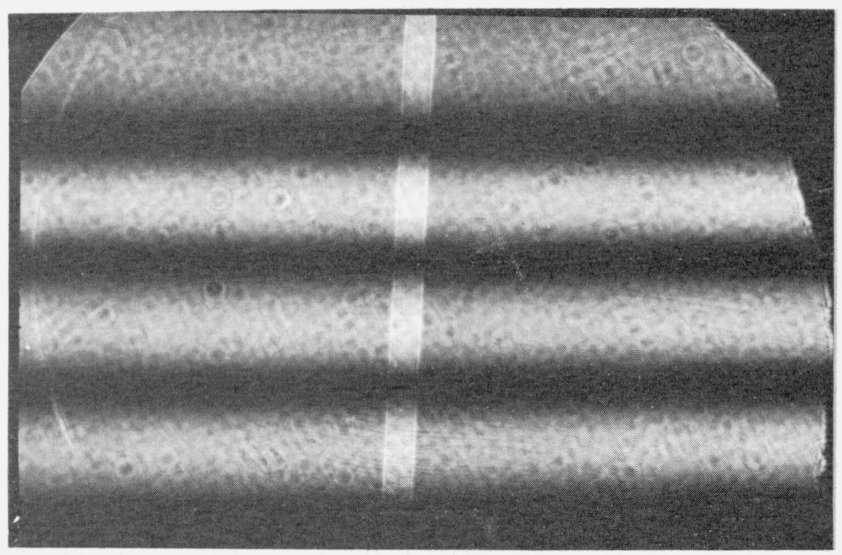

FIGURE 7. Typical fringe pattern obtained in vacuum.

Arrangement as in figure l(b), motor speed $90 \mathrm{rps}$. The grainy structure is due to inhomogeneities of the vacuum chamber window.

\section{Conclusions}

The result of the above experiment is consistent with the constant velocity of light. A ballistic theory of light is now restricted to the following possibilities:

(1) There would be no fringe-shift in our experiment if air, at a pressure of less than $10^{-6}$ torr, were capable of reradiation; to disprove this possibility, the present or an equivalent experiment would have to be performed in a vacuum satisfying (6a).

(2) There would also be no fringe-shift if the Lloyd mirror, instead of reradiating the incident light, were to reflect it without change in velocity (the velocity of both rays would then be the same). This could be due to the special case of grazing incidence occurring in this interferometer, or else this could be a general law of reflection. As far as we are aware, only two direct experiments have ever been performed to investigate the velocity of light from a moving mirror: one by Michelson [1913], the other by Majorana [1918, 1919]. Neither found a deviation from the value $c_{0}$, but both experiments were performed in air at atmos- 
pheric pressure. Thus (5) could still be consistent with all experimental observations if light were transmitted with a velocity depending on that of the medium only (e.g., by reradiation), but reflected with a velocity dependent on the incident velocity (e.g., analogously to tennis balls bouncing off a wall).

Both of these possibilities could be tested by repeating the experiments by Michelson [1913] and Rotz [1963] in a vacuum high enough to satisfy (6a). It is admittedly doubtful whether either of these experiments will contradict (4); however, their outcome is not absolutely certain, and considering what is at stake, we consider further experiments to prove (4) by direct measurement worthwhile.

The above investigation was made possible by a grant of the Council on Research and Creative Work of the University of Colorado. Every possible assistance was given to us by Frank S. Barnes. We are particularly indebted to Ball Bros., Boulder, Colo., who made their large vacuum chamber available for the experiment, and for the assistance rendered by their collaborators M. Folk, N. Gossett, and R. Johnson. J. Pierce was most cooperative in machining the parts of the apparatus and Mrs. Charlotte I. Cranford typed the manuscript.

One of us (P. Beckmann) had already performed the experiment (in air) at the Institute of Radio Engineering, Czechoslovak Academy of Sciences, Prague, in Summer, 1963; he was assisted by several persons working far beyond their ordinary duties, in particular, J. Eigl, E. Martinec, and V. Polášek of the V.U.Z.O.R.T. Institute, R. Lanc and G. Pressburger of the URECSAV Institute, and many others. Although for lack of time no conclusive result was then obtained, the experience gained was applied in the present experiment.

We are grateful to J. P. Neal of the NBS, Boulder, Colo., for having drawn our attention to an embarrassing numerical error in equation (6).

\section{References}

Babcock, G. C., and T. G. Bergman (1964), Determination of the constancy of the speed of light, J. Opt. Soc. Am. 54, 147-151.

Beckmann, P., and P. Mandics (1964), Experiment on the constancy of the velocity of electromagnetic radiation, Radio Sci. J. Res. NBS/USNC/URSI 68D, No. 12, 1265-1268.

Bonch-Bruevich, M. A., and V. A. Molchanov (1956), A new optical relativistic experiment, (in Russian) Opt. i Spektr. 1, 113-124.

Born, M., and E. Wolf (1959), Principles of Optics, pp. 100-103, (Pergamon Press, London-New York).

Dingle, H. (1959), A proposed test of the "ballistic" theory of light, Month. Not. Roy. Astr. Soc. 119, 67-71.

Dingle, H. (1960a), Relativity and electromagnetism: an epistemological appraisal, Phil. of Sc. 27, 233-253.

Dingle, H. (1960b), The origin and present status of the special relativity theory, Science Progress 48, 201-219.

Dingle, H. (1960c), The Doppler effect and the foundations of physics, Br. J. Phil. Sc. 11, 113-129.

Fox, J. G. (1962), Experimental evidence for the second postulate of relativity, Am. J. Phys. 30, 297-300.

Kantor, W. (1962), Direct first-order experiment on the propagation of light from a moving source, J. Opt. Soc. Am. 52, 978-984.

Majorana, Q. (1918), On the second postulate of the theory of relativity: an experimental demonstration of the constancy of the velocity of light reflected by a moving mirror, Phys. Rev. 11, 411-420.

Majorana, Q. (1919), Experimental demonstration of the constancy of velocity of light emitted by a moving source, Phil. Mag. 37, 145-150.

Michelson, A. A. (1913), Effect of reflection from a moving mirror on the velocity of light, Astrophys. J. 37, 190-193.

Ritz, W. (1908), Récherches critiques sur l'électrodynamique générale, Ann. Chim. et Phys. 13, 145-275.

Rotz, F. B. (1963), New test of the velocity of light postulate, Phys. Letters 7, No. 4, 252-254.

Tolman, R. C. (1910), The second postulate of relativity, Phys. Rev. 31, 26-40.

Tolman, R. C. (1912), Some emission theories of light, Phys. Rev. 35, 136-143.

Tomaschek, R. (1924), Ueber das Verhalten des Lichtes ausserirdischer Quellen, Ann. d. Phys. 73, 105-144. 